\title{
Análise de discrepâncias nas prescrições de pacientes acompanhados pela clínica cirúrgica de um hospital público
}

Discrepancy analysis of prescriptions for patients observed by the surgical clinic in a public hospital

Análisis de discrepancias en las prescripciones de pacientes seguidos en la clínica quirúrgica de un hospital público

Simona Renz Baldin

ORCID: https://orcid.org/0000-0001-7825-0462 Universidade Estadual do Oeste do Paraná, Brasil E-mail: simona.renz@hotmail.com

Fabiana Sari Ferreira

ORCID: https://orcid.org/0000-0002-2358-6919 Universidade Estadual do Oeste do Paraná, Brasil E-mail: sari.fabiana@gmail.com

Ana Flávia Redolfi Oliota

ORCID: https://orcid.org/0000-0002-3541-3484 Universidade Estadual do Oeste do Paraná, Brasil E-mail: anaoliota@gmail.com

Fabiola Giordani

ORCID: https://orcid.org/0000-0003-2919-856X Universidade Federal Fluminense, Brasil E-mail: fabiolagiordani@id.uff.br

Patricia Guerrero de Sousa

ORCID: https://orcid.org/0000-0002-2395-9463 Universidade Estadual do Oeste do Paraná, Brasil E-mail: patrícia.guerrero@ unioeste.br Luciana de Fátima Caldeira ORCID: https://orcid.org/0000-0002-9042-8689 Universidade Estadual do Oeste do Paraná, Brasil E-mail: luciane-caldeira@hotmail.com

Andréia Cristina Conegero Sanches ORCID: https://orcid.org/0000-0002-3108-7300 Hospital Universitário do Oeste do Paraná, Brasil E-mail: andreiaconegero@gmail.com

\begin{abstract}
Resumo
O uso inapropriado de um medicamento pode ocasionar danos e problemas de saúde, neste contexto a conciliação de medicamentos é um processo que visa identificar identificar e comparar os medicamentos de uso domiciliar para garantir melhor assistência ao paciente. Neste contexto, o presente estudo teve como objetivo comparar discrepâncias, tanto as intencionais, quanto as não-intencionais encontradas nos pacientes admitidos na clínica cirúrgica de um hospital público. Através de entrevista estruturada, realizada antes da admissão e comparada com as prescrições após a admissão. A classificação das discrepâncias foi em intencionais e não-intencionais. Em cem (100) pacientes analisados, cinquenta e nove (59) apresentaram alguma discrepância. Desses, a maioria era do sexo feminino (68\%), com mediana de idade de 59 anos. Das cinquenta e sete (57) discrepâncias não-intencionais encontradas, $78,9 \%$ ocorreram por omissão, e 15,8\% por dose. Já as intencionais foram sessenta e seis (66), sendo $84,9 \%$ por omissão, devido a protocolos estabelecidos pelo hospital. Sendo assim, o estudo demonstra uma alta taxa de discrepâncias encontradas nos pacientes, sugerindo a importância da implantação da conciliação realizada pelo farmacêutico visando reduzir o número de problemas relacionados à farmacoterapia, para garantir a segurança do paciente.
\end{abstract}

Palavras-chave: Erros de medicação; Prescrição inapropriada; Segurança do paciente; Reações adversas e efeitos colaterais relacionados a medicamentos.

\section{Abstract}

Health problems can be caused by improper use of medicines, in this context, a reconciliation of medicines is a process that aims to identify and compare medicines for home use to ensure a better treatment to the patient. In this 
context, the present study aimed to compare intentional and unintentional discrepancies found in patients prescriptions admitted to the surgical clinic of a public hospital. Through a structured interview, carried out before admission and compared with the prescriptions after admission. Discrepancies were classified as intentional and unintentional. In one hundred (100) patient patients, fifty-nine (59) independent discrepancies. Of these, the majority were female $(68 \%)$, with a median age of 59 years. Of the fifty-seven (57) unintentional discrepancies found, $78.9 \%$ occurred by default, and $15.8 \%$ per dose. The intentional ones were sixty-six (66), being $84.9 \%$ by default, due to protocols prepared by the hospital. Thus, the study demonstrates a high rate of discrepancies found in patients, suggesting the importance of implementing a pharmacist reconciliation that reduces the number of problems related to pharmacotherapy, to ensure patient safety.

Keywords: Medication errors; Inappropriate prescribing; Patient safety; Drug-related side effects and adverse reactions.

\section{Resumen}

El uso indebido de un medicamento puede ocasionar daños y problemas de salud, en este contexto la conciliación de medicamentos es un proceso que tiene como objetivo identificar y comparar medicamentos de uso domiciliario para asegurar la mejor atención al paciente. En este contexto, el presente estudio tiene como objetivo comparar las discrepancias intencionales y no intencionales encontradas en pacientes ingresados en la clínica quirúrgica de un hospital público. Mediante entrevista estructurada, realizada antes del ingreso y comparada con las prescripciones posteriores al ingreso. Las discrepancias se clasificaron como intencionales y no intencionales. En cien (100) pacientes analizados, cincuenta y nueve (59) presentaron alguna discrepancia. De estos, la mayoría eran mujeres (68\%), con una edad media de 59 años. De las cincuenta y siete (57) discrepancias no intencionales encontradas, el $78,9 \%$ se producen por defecto y el $15,8 \%$ por dosis. Las intenciones fueron sesenta y seis (66), siendo el $84,9 \%$ defectuoso, debido a los protocolos establecidos por el hospital. Así, el estudio demostró una alta lista de discrepancias encontradas entre los pacientes, lo que sugiere la importancia de implementar la conciliación realizada por el farmacéutico con el fin de reducir el número de problemas relacionados con la farmacoterapia, para garantizar la seguridad del paciente.

Palabras clave: Errores de medicación; Prescripción inadecuada; Seguridad del paciente; Efectos colaterales y reacciones adversas relacionados con medicamentos.

\section{Introdução}

A transição entre os diferentes níveis de atenção é um dos pontos críticos na assistência hospitalar. Durante este processo, pode ocorrer uma falha na comunicação entre os profissionais de saúde, pacientes e familiares, determinando a perda de informações essenciais, o que possibilita o acontecimento de erros de medicação, que comprometem a segurança do paciente (Coleman \& Berenson, 2004; Bodenheimer, 2008; Kwan, Sampson \& Shojania). Desta forma, entende-se por erros de medicação qualquer evento que leve o paciente ao uso inapropriado de um medicamento que pode ocasionar danos e problemas de saúde (Anacleto et al., 2010).

Os erros de medicação, juntamente com os danos causados ao paciente também podem ser resultados de um histórico de medicação coletados de forma incompleta ou imprecisa e, é uma das principais causas de morbidade entre os pacientes admitidos no hospital. Durante a admissão hospitalar, o processo adequado de coleta do histórico de medicação que o paciente já faz uso bem como sua situação clínica detalhada são importantes para evitar discrepâncias (Hayes et al., 2007).

As discrepâncias são definidas como "qualquer diferença entre a medicação usada pelo paciente antes da admissão e a medicação prescrita no momento da admissão hospitalar" (Pippins et al., 2008). Além disso, as discrepâncias são divididas em intencionais e não-intencionais; e vale salientar que quando a discrepância não é intencional, são considerados erros de medicação, que podem ter consequências clínicas e causar danos ou ter potencial para causar danos. Discrepâncias nãointencionais são as mais comuns em causarem eventos adversos reais ou potenciais e são fáceis de serem evitados (Vira, Colquhoun \& Etchells, 2006).

A conciliação de medicamentos é um processo que visa identificar e comparar todos os medicamentos que o paciente fazia uso em domicílio, bem como compará-los com aqueles prescritos na internação. Esse processo detecta possíveis erros, inconsistências e interações medicamentosas, os quais possibilitam melhor assistência ao paciente (Anacleto et al., 2010). 
Assim, os objetivos deste trabalho foram avaliar e classificar as discrepâncias identificadas na farmacoterapia de pacientes bem como traçar o perfil desses e correlacionar fatores que puderam influenciar no quantitativo das discrepâncias encontradas na farmacoterapia durante a admissão na clínica cirúrgica de um hospital público no Estado do Paraná.

\section{Metodologia}

Trata-se de um estudo transversal, quantitativo e prospectivo (Pereira, 2018), realizado em um hospital universitário, público, de uma cidade no Oeste do Paraná, Brasil, com 266 mil habitantes. Fizeram parte da amostra por conveniência todos os pacientes internados no pronto socorro e os da ala cirúrgica, atendidos pela equipe de clínica cirúrgica, de origem externa à instituição, durante os meses de fevereiro a maio de 2018.

Neste estudo, foram incluídos pacientes maiores de 18 anos, de ambos os sexos, internados na unidade de pesquisa, que faziam uso de medicamentos contínuos e que estavam de acordo com os critérios apresentados no Termo de Consentimento Livre e Esclarecido (TCLE). Pacientes foram excluídos quando: não houve a possibilidade de realizar a entrevista em 48 horas, receberam alta antes de serem entrevistados, já haviam participado do estudo anteriormente devido à internação prévia, eram acompanhados por outros serviços farmacêuticos do hospital ou possuíam algum problema cognitivo e/ou problema de fala, sem acompanhantes/cuidador.

Os dados foram coletados diariamente por entrevista com o paciente e a análise de prontuário no momento da internação. Por meio de ficha previamente estruturada utilizada na entrevista com o paciente, foi obtido o histórico de uso de medicamentos. Informações também foram obtidas das prescrições médicas, da análise dos medicamentos trazidos de casa pelo paciente e na entrevista com os familiares ou cuidador. Os dados foram organizados em uma planilha que continha uma lista dos medicamentos usados até o momento da admissão e outra com os medicamentos que foram prescritos no hospital. As discrepâncias foram identificadas a partir da comparação destas listas (Cornish et al., 2005; Lombardi et al., 2016).

De acordo com Gleason et al. (2010) e Zoni et al (2012) foi realizado a classificação das discrepâncias, sendo consideradas intencionais quando havia mudança na condição do paciente, medicamentos de uso esporádico/sintomático, protocolos institucionais ou justificativa pontual. Com o não-intencionais, foram os casos de omissão, duplicidade, divergência de dose, frequência, intervalo ou via de administração.

As análises estatísticas foram realizadas com auxílio do software SPSS, versão 13.0, da empresa IBM. As variáveis contínuas foram descritas usando média e desvio padrão ou mediana e intervalo interquartílico (IQ), dependendo da natureza da distribuição dos dados. As frequências foram utilizadas para descrever as variáveis categóricas.

A comparação entre as variáveis contínuas com e sem discrepância foi realizada pelo teste t para dados que foram normalmente distribuídos ou o Mann-Whitney para dados não paramétricos na comparação das médias. O teste de quiquadrado ou a fórmula corrigida de Yates foi utilizada para variáveis categóricas visando estabelecer correlações entre as variáveis. Todos os testes foram considerados significativos com o $\mathrm{p}<0,05$.

Os medicamentos foram classificados de acordo com a classificação anatômica terapêutica química (Anatomical Therapeutical Chemical - ATC), segundo os grandes grupos sistêmicos ( $1^{\circ}$ nível), da Organização Mundial de Saúde (WHO, 2020). Foram feitas análises de correlação com as características dos pacientes, comorbidades e uso de medicamentos antes e após o internamento. O estudo foi aprovado pelo Comitê de Ética em pesquisa com seres humanos da Universidade Estadual do Oeste do Paraná (UNIOESTE), sob parecer nº 5.370.127.

\section{Resultados}

Durante o período de pesquisa, 278 pacientes foram acompanhados pela clínica cirúrgica e, desses, cem pacientes foram incluídos no estudo. Foram excluídos 178 pacientes, em que $131(73,6 \%)$ não faziam uso de medicamentos antes da 
admissão hospitalar, dezesseis (16) pacientes (9\%) apresentavam idade menor que 18 anos, dezesseis (16) (9\%) estavam internados há mais de 48 horas, treze (13) (7,3\%) devido ao isolamento, o que impossibilitava a coleta de dados e dois (2) $(1,1 \%)$ já estavam em acompanhamento por outro serviço farmacêutico (ambulatório de obesidade).

A mediana de idade foi de 59 anos, com variação interquartílica entre 20 e 84 anos, sendo $60 \%$ do sexo feminino. As informações foram colhidas diretamente com o paciente (78\%), paciente e seu familiar ou cuidador (20\%) e entrevista somente com o familiar (2\%). As informações foram obtidas nos prontuários para todos os casos. A Tabela 1 abaixo demonstra as características demográficas e hábitos de vida e de saúde dos pacientes.

Tabela 1 - Caracterização dos pacientes acompanhados pela clínica cirúrgica em um hospital público.

\begin{tabular}{|c|c|c|c|}
\hline Características & $\mathbf{N}=\mathbf{1 0 0}$ & Características & $\mathbf{N}=\mathbf{1 0 0}$ \\
\hline Sexo & & Tabagismo & \\
\hline Feminino & 60 & Sim & 11 \\
\hline \multirow[t]{2}{*}{ Masculino } & 40 & Não & 63 \\
\hline & & Ex usuário & 26 \\
\hline Idade mediana em anos (variação) & $59(20$ a 84$)$ & Etilismo & \\
\hline Alergia a medicamentos & & Sim & 21 \\
\hline Sim & 14 & Não & 65 \\
\hline Não & 86 & Ex usuário & 14 \\
\hline Alergia a alimentos & & Presença de doenças prévias & \\
\hline Sim & 12 & Sim & 83 \\
\hline Não & 88 & Não & 17 \\
\hline Uso de chás & & Reação adversa a medicamentos & \\
\hline Sim & 77 & Sim & 7 \\
\hline Não & 23 & Não & 93 \\
\hline
\end{tabular}

Fonte: Dados dos autores.

Todos os pacientes incluídos no estudo foram admitidos para cirurgias gerais sendo 39\% devido a hérnia, $24 \%$ colelitíases, $11 \%$ dor abdominal/abdome agudo e $26 \%$ outros motivos.

O tempo de internamento apresentou mediana de três dias, com variação interquartílica entre 2 e 22 dias. Verificouse que oitenta e três (83) pacientes apresentaram alguma doença prévia, conforme mostra o Gráfico 1, abaixo. 
Gráfico 1 - Frequência das doenças prévias dos pacientes acompanhados pela clínica cirúrgica em um hospital público.

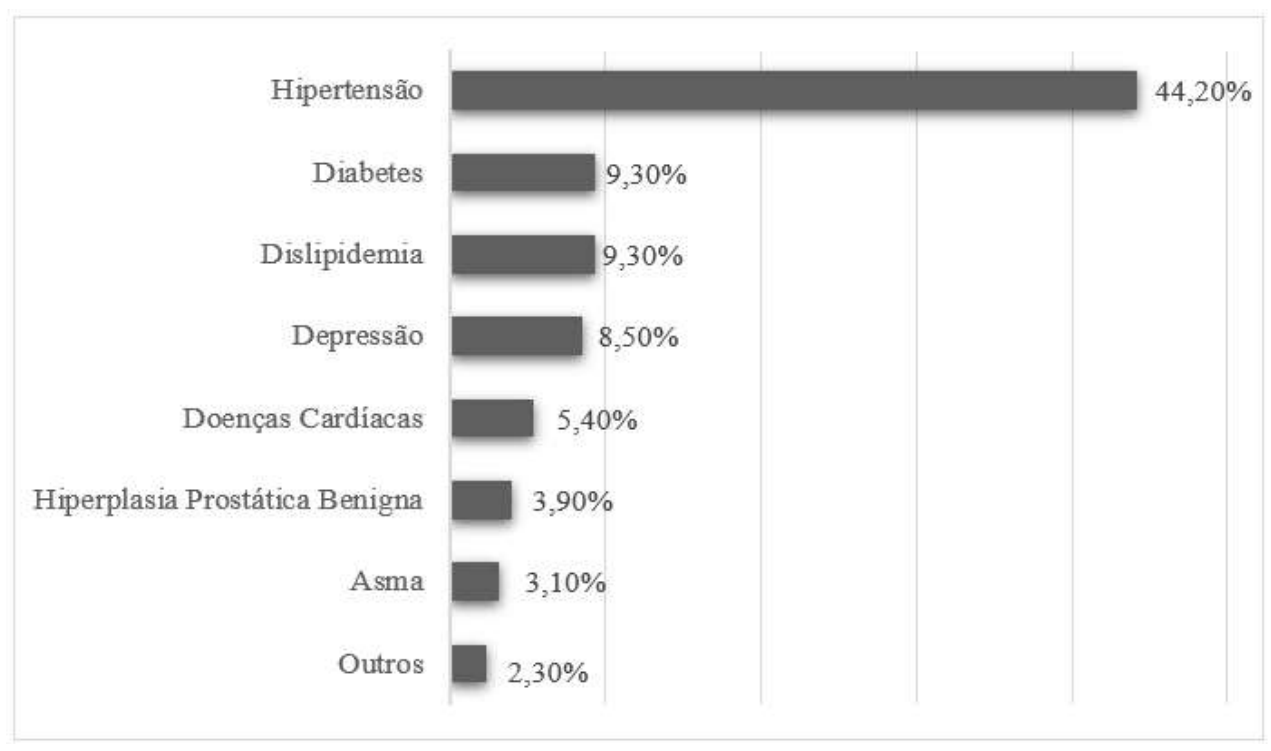

Fonte: Dados dos autores.

Quanto ao uso de tabaco, $11 \%$ dos pacientes eram fumantes, $63 \%$ nunca fizeram uso de tabaco e $26 \%$ dos pacientes declararam abandono do hábito. Sobre o consumo de bebidas alcoólicas, $21 \%$ faziam uso frequente, $65 \%$ nunca consumiram bebidas alcóolicas e $14 \%$ deixaram de usar.

Na pré-admissão, verificou-se a utilização média de 3,15 + 2,39 medicamentos/paciente, com variação mínima de um (1) e máxima de doze (12) medicamentos. Porém, com os medicamentos após a internação foi de 2,09 + 3,10 medicamentos por paciente, com variação mínima de um (1) e máxima de dezenove (19) medicamentos. A média de medicamentos utilizados por faixa etária segue na tabela abaixo.

Entre os pacientes avaliados, observou-se que 59\% apresentaram pelo menos uma discrepância, sendo que 22 (37,3\%) pacientes apresentaram discrepâncias não-intencionais, 27 (45,8\%) discrepâncias intencionais e 10 (16,9\%) pacientes apresentaram ambas. A Tabela 2 descreve as discrepâncias encontradas.

Tabela 2 - Discrepâncias identificadas no tratamento dos pacientes acompanhados pela clínica cirúrgica em um hospital público.

\begin{tabular}{|c|c|c|}
\hline & Tipos de discrepância & n (\%) \\
\hline \multirow{5}{*}{ 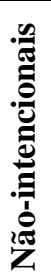 } & Omissão & $45(78,9)$ \\
\hline & Duplicidade & $1(1,8)$ \\
\hline & Dose & $9(15,8)$ \\
\hline & Intervalo/Frequência & $2(3,5)$ \\
\hline & Total & $57(100)$ \\
\hline \multirow{3}{*}{ 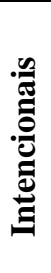 } & $\begin{array}{l}\text { Protocolos Institucionais } \\
\text { (Omissão/Suspensão) }\end{array}$ & $56(84,9)$ \\
\hline & $\begin{array}{l}\text { Justificativa pontual } \\
\text { (Mudança de dose/Posologia) }\end{array}$ & $10(15,1)$ \\
\hline & Total & $66(100)$ \\
\hline
\end{tabular}

Fonte: Dados dos autores. 
Vale ressaltar que das 123 discrepâncias encontradas 66 (53,7\%) foram intencionais e 57 (46,3\%) não-intencionais. Entre os diversos tipos de discrepância, a única que não foi identificada foi com relação à modificação da via de administração. A discrepância não intencional mais presente foi por omissão $(n=45 ; 78,9 \%)$, que ocorreu quando o paciente internou e os medicamentos de uso prévio não foram prescritos. As discrepâncias intencionais encontradas dividiram-se entre $56(84,9 \%)$ por protocolos institucionais, que ocorreram com a omissão/suspensão dos medicamentos usados previamente, e $10(15,1 \%)$ por justificativa pontual que ocorreu com mudança da dose e intervalo na tomada dos medicamentos.

Os grupos de medicamentos envolvidos nas discrepâncias encontradas, foram classificados de acordo com o sistema de classificação anatômica terapêutica química (Anatomical Therapeutical Chemical - ATC). Então, observou-se que, nas discrepâncias intencionais, as classes de medicamentos com maior frequência foram as do aparelho digestivo e metabolismo, representadas pelos medicamentos para diabetes (metformina) e antiácidos (omeprazol). Já nas discrepâncias nãointencionais, os medicamentos mais prevalentes foram do aparelho cardiovascular representado pelos anti-hipertensivos e diuréticos (metildopa, atenolol e hidroclorotiazida), e os hipolipemiantes (sinvastatina), seguidos do aparelho geniturinário representado pelos contraceptivos hormonais de uso sistêmico.

A relação entre as características do paciente, comorbidades e dados de uso de medicamento antes e após o internamento, na Tabela 3, permitiu avaliar a correlação entre fatores que podem contribuir na presença de discrepância. 
Tabela 3 - Fatores associados à presença de discrepância na farmacoterapia de pacientes acompanhados pela clínica cirúrgica de um hospital público.

\begin{tabular}{lccc}
\hline Característica & Com discrepâncias(n=59) & Sem Discrepâncias (n=41) & p \\
\hline Idade (anos) & $53,5(15,5)$ & $59,0(11,5)$ & 0,077 \\
Idade categorizada & & & \\
$\quad>60$ anos & $24(49 \%)$ & $25(51,0 \%)$ & $0,046^{*} 1$ \\
$\quad<60$ anos & $35(68,6 \%)$ & $16(31,4 \%)$ &
\end{tabular}

Sexo

$\begin{array}{llll}\text { Feminino } & 41(68,3 \%) & 19(31,7 \%) & 0,020 * 1 \\ \text { Masculino } & 18(45,0 \%) & 22(55,0 \%) & \end{array}$

Alergia a medicamentos

$\begin{array}{lccc}\text { Sim } & 7(50 \%) & 7(50 \%) & 0,460 \\ \text { Não } & 52(60,5 \%) & 34(39,5 \%) & \end{array}$

Alergia a alimentos

$\begin{array}{lccc}\text { Sim } & 7(58,3 \%) & 5(41,7 \%) & 0,960 \\ \text { Não } & 52(59,1 \%) & 36(40,9 \%) & \end{array}$

Uso de chás

$\begin{array}{lccc}\text { Sim } & 44(57,1 \%) & 33(42,9 \%) & 0,490 \\ \text { Não } & 15(65,2 \%) & 8(34,8 \%) & \end{array}$

Tabagismo

$\begin{array}{lccc}\text { Sim } & 6(54,5 \%) & 5(45,5 \%) & \\ \text { Não } & 36(57,1 \%) & 27(42,9 \%) & 0,734 \\ \text { Ex usuário } & 17(65,4 \%) & 9(34,6 \%) & \end{array}$

Etilismo

$\begin{array}{lccr}\text { Sim } & 14(66,7 \%) & 7(33,3 \%) & \\ \text { Não } & 38(58,5 \%) & 27(41,5 \%) & 0,611 \\ \text { Ex usuário } & 7(50 \%) & 7(50 \%) & \end{array}$

Presença de doenças prévias

$\begin{array}{lccc}\text { Sim } & 47(56,6 \%) & 36(43,4 \%) & 0,286 \\ \text { Não } & 12(70,6 \%) & 5(29,4 \%) & \end{array}$

Reação adversa a medicamentos

$\begin{array}{lccc}\text { Sim } & 7(100 \%) & 0(0 \%) & 0,039^{2} \\ \text { Não } & 52(55,9 \%) & 41(44,1 \%) & 0,591 \\ \text { internamento } & 4,4(3,1) & 5,0(3,7) & 0,009^{1} \\ \text { dicamentos pré-admissão } & 3,8(2,7) & 2,3(1,4) & 0,569\end{array}$

Legenda: ${ }^{1}$ Resultado significativos com teste de qui-quadrado; ${ }^{2}$ Resultado significativo com teste de exato de Fisher's. Fonte: Dados dos autores.

Quando comparados os indivíduos que apresentaram discrepância com aqueles que não apresentaram, os fatores que 
contribuíram para este resultado foram: sexo feminino $(\mathrm{p}=0,020)$, idade $<60$ anos $(\mathrm{p}=0,046)$, ter apresentado reação adversa a medicamento $(p=0,039)$ e a quantidade média de medicamentos na pré-admissão $(p=0,009)$. Tais comparações foram estatisticamente significativas, segundo o teste qui-quadrado, Mann-Whitney e teste de exato de Fisher's, aplicados aos dados.

\section{Discussão}

Este estudo buscou avaliar as prescrições, identificar a presença das discrepâncias e classificar os pacientes atendidos pela clínica cirúrgica de um hospital público do Oeste do Paraná.

Características semelhantes como idade (a maioria com menos de 60 anos), sexo feminino, pacientes que relataram apresentar reação adversa a algum medicamento e que tomavam maior número de medicamentos antes do internamento (aproximadamente quatro medicamentos ou mais) foram observadas em pacientes que apresentaram discrepâncias. As correlações entre o número de discrepâncias e o sexo dos pacientes $(p=0,020)$, a idade $(p=0,046)$ e reações adversas a medicamentos $(\mathrm{p}=0,039)$ são estatisticamente significativas. Tais características corroboram com estudos anteriores (Salanitro et al., 2012; Davies et al., 2009; Nagao-Dias et al., 2004).

Os dados também foram observados no estudo de Kiechle et al. (2018), que avaliaram 1014 participantes, com 60\% do sexo feminino e $36 \%$ estavam na faixa etária entre 40 e 59 anos.

Conforme demonstrado neste estudo, $14 \%$ relataram apresentar alergia a medicamentos e $12 \%$ a alimentos. . Neste estudo realizado em 2018 com 1014 pacientes, também demonstrou que 56\% dos pacientes relataram ter alguma reação alérgica a medicamentos e desses $41 \%$ apresentaram algum tipo de discrepância (Kiechle et al., 2018).

Neste estudo, constatou-se que $77 \%$ dos participantes utilizavam chás para tratar alguma doença. Tais informações corroboram com as obtidas em um estudo realizado na região Sul do Brasil, onde se observou que 57\% dos idosos participantes utilizavam algum tipo de chá (25) (Flores \& Mengue, 2005). Já um estudo realizado na Noruega (2013) demonstrou a prevalência de 44\% dos pacientes que utilizavam chás e, em 2007 nos Estados Unidos, quase 20\% da população utilizam chás, e $41 \%$ dos adultos relataram o uso de produtos naturais para o auto tratamento antes de procurar um médico (Djuv, Nilsen \& Steinsbekk, 2013; Barnes \& Bloom, 2008; Van Der Luit et al., 2018).

O tabagismo é reconhecido como um dos maiores problemas de saúde pública não apenas entre jovens, mas entre idosos também. Este é um risco adicional para várias doenças, entre elas, as cardiovasculares que são as primeiras causas indiretas de morte mundial (Marinho et al., 2010). O consumo nocivo do álcool é um fator causal para mais de 200 tipos de doenças e lesões, incluindo doenças mentais e comportamentais, além de doenças não transmissíveis e lesões.

No que tange ao consumo de tabaco e álcool, neste estudo, $11 \%$ e $21 \%$ dos pacientes em estudo fazem uso, respectivamente. Em 2017, 21\% da população mundial adulta, ou seja, 950 milhões de homens e 177 milhões de mulheres são tabagistas e a prevalência estimada para uso de álcool foi de 35,4\% (12,4\% para mulheres e 65\% para homens) (Silva et al., 2017).

Neste estudo, a mediana no tempo de internamento foi de três dias (variando de 2 a 22 dias), isso é explicado devido a maioria dos pacientes terem realizado cirurgias eletivas. O curto tempo de internação acontece para evitar infecções hospitalares, pois representam as complicações mais frequentes no pós-operatório e elevam a chance de mortalidade. De acordo com Silva de Sousa et al. (2012), que realizaram um estudo em um hospital público da região central de São Paulo em pacientes submetidos a cirurgias em várias especialidades, o tempo de permanência na internação pós-cirúrgica para procedimentos de pequeno a médio porte foi de quatro dias.

O uso de medicamentos contínuos no domicílio demonstra que mesmo diante de toda tecnologia à disposição da medicina, os medicamentos ainda são os mais utilizados para tratar as doenças (WHO, 2002). Conforme os dados obtidos 
nesse estudo, o número de medicamentos na pré-admissão dos pacientes que apresentaram discrepâncias foi maior do que os pacientes que não apresentaram tais características. Isso demonstra que quanto mais medicamentos o paciente faz uso em casa, maiores são as chances de apresentar discrepâncias na admissão hospitalar. O mesmo resultado foi obtido em uma revisão sistemática, na qual se demonstrou que um maior número de medicamentos foi associado a uma maior taxa de discrepância (Tran et al., 2019). A frequência encontrada nesse estudo foi de 2,08 discrepâncias por admissão com pelo menos uma discrepância de medicação. Todavia, em estudos realizados por Bandrés et al. (2013), a frequência foi de 1,8, enquanto Van Der Luit et al. (2018) apresentaram 1,7 como resultado de pesquisa. Essa situação demonstra a necessidade de monitoramento para evitar inconsistências nas prescrições e garantir a segurança do paciente (Coleman et al., 2005).

Em relação às discrepâncias, as intencionais (53,7\%) foram mais frequentes que as não-intencionais (46,3\%), isso se explica devido aos protocolos estabelecidos de suspensão de algumas classes de medicamentos no momento da internação; e, no caso deste estudo, a maioria foi hipoglicemiante oral. Das discrepâncias não-intencionais encontradas, a omissão foi a predominante seguida de dose. Mendes et al. (2016) encontraram 47,4\% de discrepâncias intencionais e 52,6\% de discrepâncias não-intencionais e observaram que 59,9\% delas foram de omissão. Uma revisão sistemática, que incluiu vinte e dois estudos, demonstrou que 10 a $61 \%$ dos pacientes apresentam pelo menos um erro de omissão. Enquanto $41 \%$ de todos os erros foram clinicamente importantes e 22\% tiveram potencial para causar algum dano (Fitzgerald, 2009).

Em um estudo realizado por Kiechle et al. (2018), observou-se uma alta taxa de discrepância por omissão (40\%). Tal omissão pode ser explicada pela dificuldade dos pacientes ou do cuidador em relatar os medicamentos usados em casa. Os pacientes são vulneráveis na admissão e na alta hospitalar, e as discrepâncias foram relatadas como responsáveis por mais da metade dos erros de medicação (Rozich et al., 2004). A omissão de medicamentos pode causar a interrupção do tratamento e, consequentemente, danos ao paciente (Gleason et al., 2010).

Sobre os medicamentos mais envolvidos em discrepâncias, diversos autores relataram que a maioria fazia parte do aparelho cardiovascular (Bandres et al., 2013; Moriel et al., 2008; Lehnbom et al., 2014; Hias et al., 2017), seguidos do trato alimentar e metabolismo (Hias et al., 2017; Beckett, Crank \& Wehmeyer, 2012). Estas informações corroboram com os dados encontrados que demonstraram que as doenças mais frequentes foram relacionadas ao sistema cardiovascular, aparelho digestivo e metabolismo.

Uma revisão sistemática demonstrou que erros no histórico de medicação do paciente na admissão hospitalar mostraram que 34 a 95\% dos pacientes que apresentaram pelo menos uma discrepância tinha alergias e/ou reação adversa a medicamentos (Tran et al., 2019).

Pesquisas mostram que algumas estratégias como a obtenção da melhor história possível da medicação, o envolvimento de uma equipe multiprofissional e principalmente o farmacêutico foram úteis para a redução de discrepâncias, o processo de reconciliação de medicamentos e processos de documentação de alergias (Johnston, Saulnier \& Gould, 2010).

A presença do farmacêutico no setor contribui para a detecção de discrepâncias que poderiam passar desapercebidas pelo prescritor e poderiam causar prejuízo ao paciente. Isso mostra que os pacientes em ambiente hospitalar necessitam de acompanhamento multiprofissional para garantir maior segurança durante o uso de medicamentos.

Neste estudo, algumas limitações devem ser consideradas, tais como o número pequeno de participantes, devido à alta rotatividade de pacientes no setor (clínica cirúrgica). A presença do farmacêutico no setor, mesmo que por pouco tempo, foi outro fator importante e pode ter influenciado positivamente na conduta do prescritor e, consequentemente, no total de discrepâncias encontradas, pois os médicos e residentes, ao tomarem conhecimento do estudo, podem ter sido motivados a se preocuparem mais com as prescrições e históricos dos medicamentos dos pacientes, levando ao efeito de Hawthorne (Sousa \& Mendes, 2014).

Apesar das interferências, acredita-se que o resultado possa contribuir para mostrar os benefícios do olhar atento do 
farmacêutico na farmacoterapia do paciente bem como gerar dados para futuras comparações. Assim como, poder garantir a maior segurança na assistência ao paciente e gerar indicadores de qualidade para o gerenciamento das melhorias no serviço prestado (Ferreira et al, 2021).

\section{Conclusão}

Nesse estudo, foi traçado o perfil das discrepâncias encontradas, constatando-se um número significativo de discrepâncias não-intencionais, e a omissão foi a mais frequente. Tal fato causa grande preocupação devido à chance de causar danos e eventos adversos que podem ser evitáveis.

Com esses resultados, demonstra-se que é necessário e importante a avaliação das discrepâncias, pela implantação do serviço de conciliação de medicamentos como uma prática organizacional e estratégica destinada a assegurar que os medicamentos usados antes do internamento sejam mantidos, quando necessários, a fim de que sejam reduzidos os erros de medicação e riscos ao paciente e, para que haja uma conciliação de alta que garanta a farmacoterapia do paciente.

O serviço prestado pelo profissional farmacêutico junto da equipe médica para realização da conciliação medicamentosa é de notável importância e por isso, sugerimos que sejam realizados treinamentos para as equipes envolvidas e posteriormente sejam comparadas as estratégias adotadas pela educação continuada com os resultados encontrados neste estudo.

\section{Referências}

Anacleto, T. A., Rosa, M. B., Neiva, H. M. \& Martins, M. A. P. (2010). Erros de medicação. Pharm Bras, 7-8.

Bandres, M. A. A., Mendoza, M. A., Nicolás, F. G., Hernandez, M. A. C. \& La Iglesia, F. R. (2013). Pharmacist-led medication reconciliation to reduce discrepancies in transitions of care in Spain. Int J Clin Pharm, 35(6), 1083-90.

Barnes, M. P. \& Bloom, B. (2008). Complementary and Alternative Medicine Use Among Adults and Children: United States.

Beckett, R. D., Crank, C. W. \& Wehmeyer, A. (2012). Effectiveness and feasibility of pharmacist-led admission medication reconciliation for geriatric patients. J Pharm Pract, 25(2), 136-41.

Bodenheimer, T. (2008). Coordinating Care - A Perilous Journey through the Health Care System. N Engl J Med, 358(10), 1064-71.

Coleman, E. A. \& Berenson, R. A. (2004). Lost in transition: Challenges and opportunities for improving the quality of transitional care. Ann Intern Med, 141(7), 533-6.

Coleman, E. A., Smith, J. D., Raha, D. \& Min, S. (2005). Posthospital Medication Discrepancies. Arch Intern Med, $165(16), 1842$.

Cornish, P. L., Knowles, S. R., Marchesano, R., Tam, V., Shadowitz, S., Juurlink, D. N. \& Etchells, E. E. (2005). Unintended Medication Discrepancies at the Time of Hospital Admission. Arch Intern Med, 165(4), 424-9.

Davies, E. C., Green, C. F., Taylor, S., Williamson, P. R., Mottram, D. R. \& Pirmohamed, M. (2009). Adverse drug reactions in hospital in-patients: A prospective analysis of 3695 patient-episodes. PLoS One, 4(2).

Djuv, A., Nilsen, O. G. \& Steinsbekk, A. (2013). The co-use of conventional drugs and herbs among patients in Norwegian general practice: A crosssectional study. BMC Complement Altern Med, 13.

Ferreira, F. S., Pereira, T. A., Souza, B. P., Sanches, A. C. C. (2021). O papel do farmacêutico na prevenção de erros de medicação. Research, Society and Development, 10(3).

Fitzgerald, R. J. (2009). Medication errors: The importance of an accurate drug history. Br J Clin Pharmacol, 67(6), 671-5.

Flores, L. M. \& Mengue, S. S. (2005). Uso de medicamentos por idosos em região do sul do Brasil. Rev Saude Publica, 39(6), 924-9.

Gleason, K. M., McDaniel, M. R., Feinglass, J., Baker, D. W., Lindquist, L., Liss, D. \& Noskin, G. A. (2010) Results of the medications at transitions and clinical handoffs (match) study: An analysis of medication reconciliation errors and risk factors at hospital admission. J Gen Intern Med. $25(5): 441-7$.

Hayes, B. D, Donovan, J. L., Smith, B.S. \& Hartman, C. A. (2007). Pharmacist-conducted medication reconciliation in an emergency department. Am J Heal Pharm, 64(16), 1720-3.

Hias, J., Van der Linden, L., Spriet, I., Vanbrabant, P., Willems, L., Tournoy, J. \& Winter, S. (2017). Predictors for unintentional medication reconciliation discrepancies in preadmission medication: a systematic review. Eur J Clin Pharmacol, 73(11),1355-77. 
Johnston, R., Saulnier, L. \& Gould, O. (2010). Best possible medication history in the emergency department: Comparing pharmacy technicians and pharmacists. Can J Hosp Pharm, 63(5), 359-65.

Kiechle, E. S., McKenna, C. M., Carter, H., Zeymo, A., Gelfand, B. W., DeGeorge, L. M., Sauter, D. A. \& Mazer-Amirshahi, M. (2018). Medication Allergy and Adverse Drug Reaction Documentation Discrepancies in an Urban, Academic Emergency Department. J Med Toxicol, 14(4), $272-7$.

Kwan, J. L., Lo, L., Sampson, M. \& Shojania, K.G. (2013). Medication Reconciliation During Transitions of Care as a Patient Safety Strategy A Systematic Review The Problem. 158(5), 397.

Lehnbom, E. C., Stewart, M. J., Manias, E. \& Westbrook, J. I. (2014). Impact of Medication Reconciliation and Review on Clinical Outcomes. Ann Pharmacother, 48(10), 1298-312.

Lombardi, N. F., Mendes, A. E. M., Lucchetta, R. C., Reis, W. C. T., Fávero, M. L. D. \& Correr, C. J. (2016). Análise das discrepâncias encontradas durante a conciliação medicamentosa na admissão de pacientes em unidades de cardiologia: Um estudo descritivo. Rev Lat Am Enfermagem, 24.

Marinho, V., Laks, J., Coutinho, E. S. F. \& Blay, S. L. (2010). Tobacco use among the elderly: a systematic review and meta-analysis. Cad Saude Publica, 26(12), 2213-33.

Mendes, A. E., Lombardi, N. F., Andrzejevski, V. S., Frandoloso, G., Correr C. J. \& Carvalho M. (2016). Medication reconciliation at patient admission: A randomized controlled trial. Pharm Pract (Granada), 14(1), 1-7.

Moriel, M. C., Pardo, J., Catalá, R. M. \& Segura, M. (2008). Prospective study on conciliation of medication in orthopaedic patients. Farm, 32(2), 65-70.

Nagao-Dias, A. T., Barros-Nunes, P., Coelho, H. L. L \& Solé, D. (2004). Reações alérgicas a medicamentos. J Pediatr (Rio J), 80(4), $259-66$.

Pereira, A. S., Shitsuka, D. M., Parreira, F. J., \& Shitsuka, R. (2018). Metodologia da Pesquisa Científica. UFSM.

Pippins, J. R., Gandhi, T. K., Hamann, C., Ndumele, C. D., Labonville, S. A., Diedrichsen, E. K., Carty, M. G., Karson, A. S., Bhan, I., Coley, C. M., Liang, C. L., Turchin, A., McCarthy, P. C. \& Schnipper, J. L. (2008). Classifying and predicting errors of inpatient medication reconciliation. J Gen Intern Med, 23(9), 1414-22.

Rozich, J. D., Howard, R. J., Justeson, J. M., Macken, P. D., Lindsay, M. E. \& Resar, R. K. (2004). Standardization as a mechanism to improve safety in health care. Jt Comm J Qual Saf, 30(1):5-14.

Salanitro, A. H., Osborn, C. Y., Schnipper, J. L., Roumie, C. L., Labonville, S., Johnson, D. C., Neal, E., Cawthon, C., Businger, A., Dalal, A. K. \& Kripalani, S. (2012). Effect of patient- and medication-related factors on inpatient medication reconciliation errors. J Gen Intern Med, 27(8), 924-32.

Silva de Sousa, M., Muniz, J. M., Cordeiro Silva, S., Fornari, J. V., Sena Barnabé, A., Barbosa de Deus, R. \& Ferraz, R. R. N. (2012). Indicações e Tempo de Permanência em Internação Pós-Cirúrgica em um Hospital Público da Cidade de São Paulo - SP. Rev Gestão em Sist Saúde, 01(01), 108-15.

Silva, E. F., Laste, G., Torres, R. L., Hidalgo, M. P. L., Stroher, R. \& Torres, I. L. S. (2017). Consumo de álcool e tabaco: fator de risco para doença cardiovascular em população idosa do sul do Brasil. Saúde e Desenvolv Hum, 5(1), 23.

Sousa, P. \& Mendes, W. (2014). Segurança do paciente: conhecendo os riscos nas organizações de saúde. Fundação Oswaldo Cruz. Editora Fiocruz.

Tran, T., Taylor, S. E., Hardidge, A., Mitri, E., Aminian, P., George, J. \& Elliott, R. A. (2019). The Prevalence and Nature of Medication Errors and Adverse Events Related to Preadmission Medications When Patients Are Admitted to an Orthopedic Inpatient Unit: An Observational Study. Ann Pharmacother, 53(3), 252-60.

Van Der Luit, C. D. E., De Jong, I. R., Ebbens, M. M., Euser, S., Verweij, S. L., Van Den Bemt, P. M. L. A., Luttikhuis, H. M. \& Becker, M. L. (2018). Frequency of occurrence of medication discrepancies and associated risk factors in cases of acute hospital admission. Pharm Pract (Granada), 16(4), 1-5.

Vira, T., Colquhoun, M. \& Etchells, E. (2006). Reconcilable differences: Correcting medication errors at hospital admission and discharge. Qual Saf Heal Care, 15(2), 122-6.

WHO - World Health Organization (2002). Policy Perspectives on Medicines - Promoting rational use of medicines: core components. World Heal Organ. http://archives.who.int/tbs/rational/h3011e.pdf

WHO - World Health Organization. Collaborating (2020) Centre for Drugs Statistics Methodology. Anatomical Therapeutic Chemical Classification (ATC Code). https://www.whocc.no/atc_ddd_index/

Zoni, A. C., Durán García, M. E., Jiménez Muñoz, A. B., Salomón Pérez, R., Martin, P. \& Herranz Alonso, A. (2012). The impact of medication reconciliation program at admission in an internal medicine department. Eur $J$ Intern Med, 23(8), 696-700. 